\title{
X-RAY MEASUREMENT OF RESIDUAL STRESSES IN TEXTURED THIN COATINGS.
}

\author{
L. DE BUYSER, P. VAN HOUTTE and E. AERNOUDT. \\ Department of Metallurgy and Materials Engineering, \\ Katholieke Universiteit Leuven, Leuven, Belgium.
}

The X-ray diffraction technique is a well known non-destructive method to determine the residual stress level in a material. The most commonly used method is the 'd- $\sin ^{2} \psi$ ' method. However for textured materials and very thin coatings $(0.1 . .2 \mu \mathrm{m})$, this method can no longer be applied as will be shown. Therefore, a new measurement method, based on the 'glance angle' technique has been developed.

\section{DESCRIPTION OF THE RESIDUAL STRESS MEASUREMENT METHOD IN VERY THIN LAYERS.}

The commonly used $d-\sin ^{2} \psi$ method cannot be applied for residual stress determination in very thin coatings. The depth of X-ray penetration in the material varies too strongly during this measurement. As a consequence, the X-rays cannot be kept totally within the thin coating. and diffraction may also occur in the substrate. Due to this, the total amount of diffracted energy in the thin layer becomes very small, and interpretation will be impossible. The only possible way to keep the radiation as much as possible within the thin film is to choose a very small value for the angle $\alpha$ of the incident beam on the surface of the sample. Further, this angle should be held constant during the measurement, in order to keep the penetration depth also constant (i.e. 'glance angle' conditions). A scheme of the expertmental set-up is shown in figure 1 .

One now has to measure the diffraction angle $2 \Theta$ of several different (hkl) planes, while keeping the sample in a fixed position $\alpha$. The angle $\alpha$ is choosen in function of the thickness of the coating and the used radiation.

\section{RESIDUAL STRESS DETERMINATION IN THE ISOTROPIC CASE.}

For good stress measurements, the goniometer equipment should be well aligned. However when using very low values for the angle $\alpha$, even a small error in alignment has a great influence on the measured 


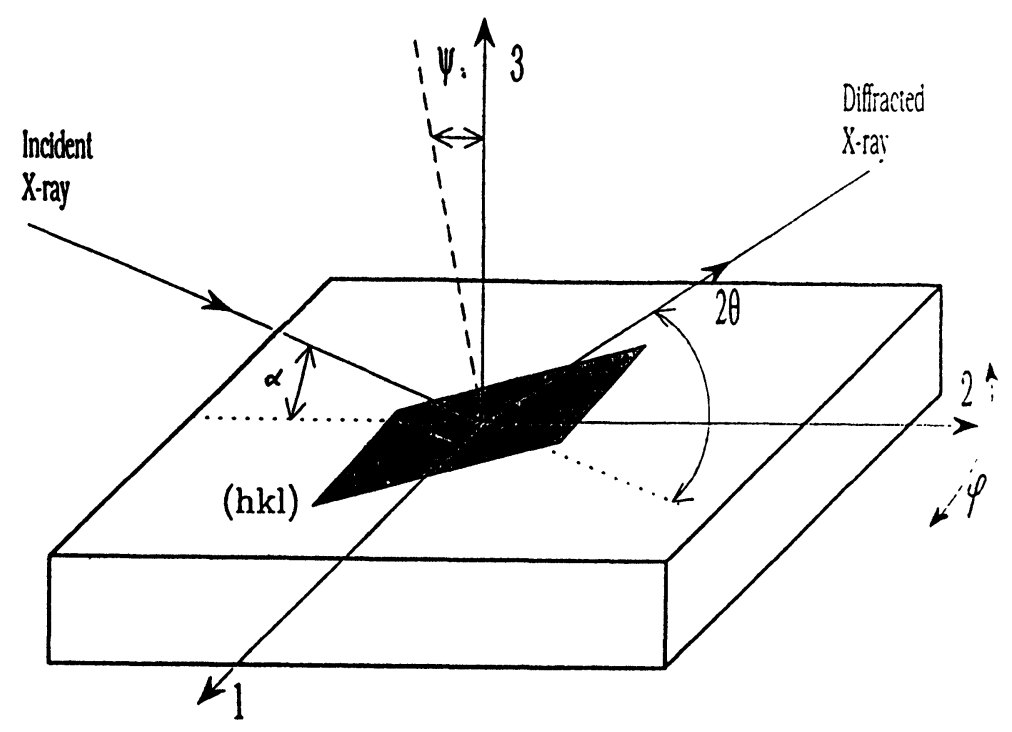

Fig.1 : Set-up for the glance angle measurement method.

data. Therefore a correction for these effects should be made. This is achieved by a second measurement on a powder sample under exactly the same measurement conditions as for the thin film. Since the stress in a powder sample can be assumed to be zero, the measured deformations can only be explained by errors in instrumental alignment. Substraction of these strains from the measured strains in the film sample, leads to:

$$
\varepsilon^{F}-\varepsilon^{P}=\varepsilon_{\sigma}+\varepsilon_{\delta}
$$

$$
\begin{aligned}
& \text { with: } \\
& \varepsilon_{\delta}
\end{aligned}=-\frac{\delta}{\text { R.sin } \alpha} \cdot \cos ^{2} \Theta
$$

$\mathrm{R}$ is the radius of the goniometer, and $\delta$ represents the shift in measurement position between the film and the powder sample (De Buyser et al [1]). The stress component $\varepsilon_{0}$, for the condition that the principal stresses $\sigma_{11}$ and $\sigma_{22}$ are equal and $\sigma_{33}=0$, is given by:

$$
\varepsilon_{\sigma}=\left(2 . S_{1}+1 / 2 S_{2}\right) \cdot \sigma \cdot\left(1-\frac{1 / 2 S_{2}}{2 \cdot s_{1}+1 / 2 S_{2}} \cdot \cos ^{2} \psi\right)
$$

$S_{1}$ and $1 / 2 S_{2}$ are the $X$-ray elastic constants, as estimated by the models of Reuss, Voigt or Hill. In figure 2 the strain $-\cos ^{2} \psi$ relation is shown for an electrochemically deposited $\mathrm{Ni}$ layer on a $\mathrm{Cu}$ substrate with a thickness of 0.6 resp. $2 \mu \mathrm{m}$. The model of equal stress (Reuss) 
was used.

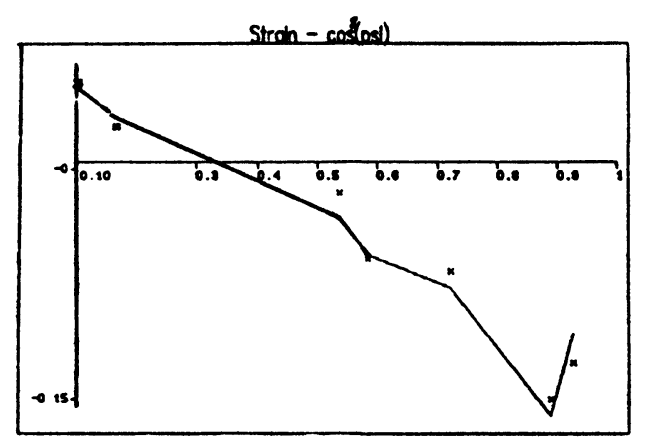

Fig. $2 a: \mathrm{Ni}: 0.6 \mu \mathrm{m}$

$$
\sigma=153 \pm 24 \mathrm{MPa}
$$

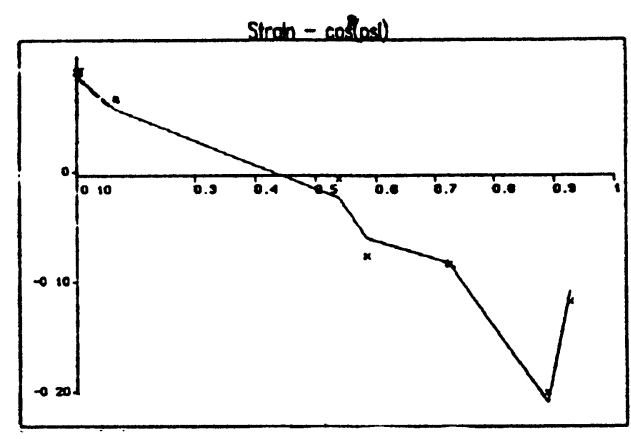

Fig. $2 b: N i: 2 \mu m$

\section{RESIDUAL STRESSES IN TEXTURED COATINGS.}

When a strong texture is present in the material, eq. (3) is no longer correct. One has to calculate new X-ray elastic constants which are orientation dependent.

\subsection{Description of the texture.}

One can describe the texture of a material using the orientation distribution function (O.D.F.). This O.D.F. is a function in 3-dimensional Euler space:

$$
\text { O.D.F. }=f\left(\varphi_{1}, \phi, \varphi_{2}\right)=f(g)
$$

where $\varphi_{1}, \phi$ and $\varphi_{2}$ are the three Euler angles, and $g$ is a symbol for these angles (Bunge [2]). The O.D.F.-calculation is based on the measurement of 4 different (hkl) pole figures. These pole figures are normally measured under the normal $\Theta / 2 \Theta$ diffraction conditions. For very thin coatings however, one again has to use glance angle conditions, with the fixed angle $\alpha$ usually much smaller than the diffraction angles $\Theta$. Since the O.D.F.-calculation assumes normal conditions, one has to transform the measured (glance angle) pole figures to satisfy this condition. This is shown in figure 3 for the (110) pole figure of iron.

One can easily see that the transformation creates a hole in the center of the polefigure, where the intensities remain unknown. This hole becomes bigger, as the difference between the incident angle $\alpha$ and 


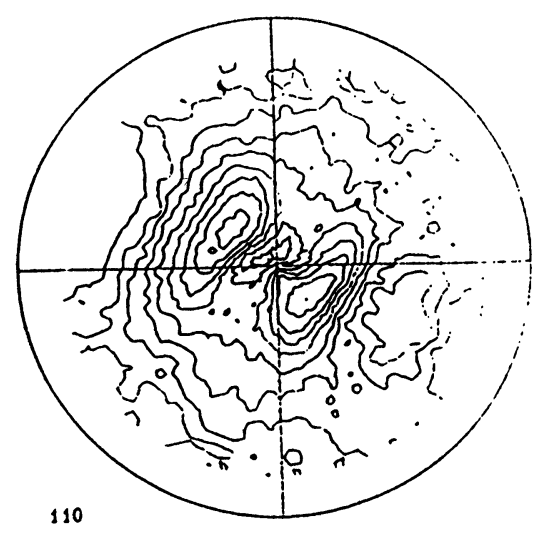

Fig.3a : (110) Pole figure of iron measured at $\alpha=14^{\circ}$.

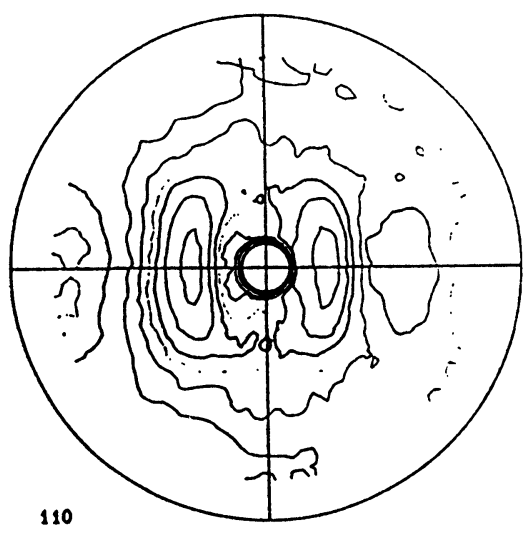

Fig.3b : Transformed version of the (110) pole figure.

the diffraction angle $\Theta$ increases. Special O.D.F.-software that takes in account the incompleteness of the pole figures in the centre of it, is currently being developed. At the time being, estimates of the unknown intensities are used. By using Mok $\alpha$ radiation the holes remain very small (because of the low diffraction angles $\Theta$ that this source delivers).

\subsection{Stress models for textured materials.}

First one has to define some reference systems. A crystal system $\{\mathrm{K}\}$ is choosen such that its axes are the [100], [010] and [001] crystal directions. The laboratory system $\{\mathrm{L}\}$ is defined by the two goniometer angles $\varphi$ and $\psi$; the axis $\mathrm{L}_{3}$ is the measuring direction. An intermediate system $\{\mathrm{I}\}$ is perpendicular to the diffracting plane. In order to calculate the X-ray elastic constants, one has to make the weighted average over all the crystal orientations that are able to diffract, i.e. varying the angle $\lambda$ from 0 to $2 \pi$ in figure 4 .

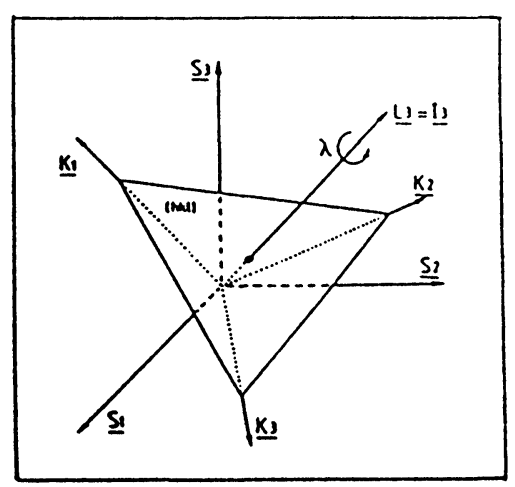

Fig. 4 : Favorably orlented crystal orientations for diffraction. 
According to the model of Reuss, this leads to:

$$
\begin{aligned}
\varepsilon_{\alpha \psi}=\varepsilon_{33}^{L} & =\frac{\int_{0}^{2 \pi} s_{33 y}^{L}(\lambda) \cdot F(g(\lambda)) \cdot d \lambda}{\int_{0}^{2 \pi} F(g(\lambda)) \cdot d \lambda} \cdot \omega_{i k} \cdot \omega_{j 1} \cdot \sigma_{k l} \\
& =f_{i y} \cdot \sigma_{i y}
\end{aligned}
$$

$\omega$ describes the transformation from the sample to the laboratory system (Serruys [3]).

According to the model of Voigt, one can find that:

$$
\varepsilon_{q \mathrm{w}}=\varepsilon_{33}^{\mathrm{L}}=\omega_{31} \cdot \omega_{3 \mathrm{j}} \cdot \mathbf{s}_{\mathrm{yk}}^{\mathrm{v}} \cdot \sigma_{\mathrm{kd}}
$$

where $\mathbf{s}^{\mathrm{v}}$ is the compliance tensor as calculated by the model of Voigt.

A combined model, where the material is considered to behave for a certain fraction $x$ like the Reuss model assumes and for a fraction (1$\mathrm{x})$ like the Voigt model, is also proposed:

$$
\varepsilon_{\text {w }}=\varepsilon_{33}^{\mathrm{L}}=\left[\mathrm{x} \cdot \mathrm{f}_{\mathrm{y}}+(1-\mathrm{x}) \cdot \omega_{3 \mathrm{k}} \cdot \omega_{31} \cdot \mathrm{s}_{\mathrm{kly}}^{\mathrm{v}}\right] \cdot \sigma_{\mathrm{l}}
$$

This model has no direct physical meaning, but can lead to a better fit of reality. The mixed model was used in figure 4 to calculate the stresses in the earlier with the isotropic model investigated coatings.

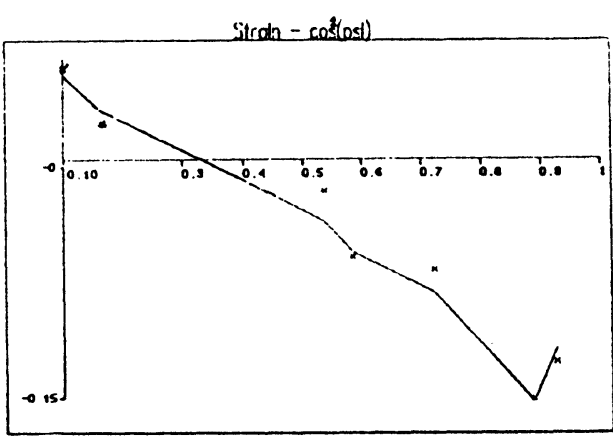

Fig.4a : Ni $0.6 \mu \mathrm{m} ; x=1$ $\sigma=165 \pm 19 \mathrm{MPa}$

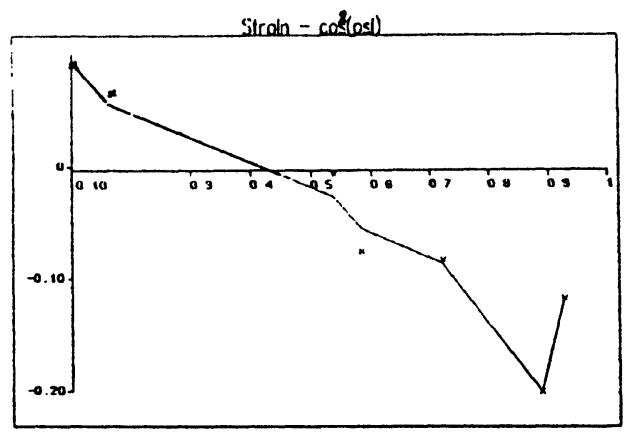

Fig. $4 b$ : Ni $2 \mu m ; x=0.8$ $\sigma=344 \pm 11 \mathrm{MPa}$

The best fit in these examples was found for $\mathbf{x}=1$ and $\mathbf{x}=0.8$ respectively for the 0.6 and $2 \mu \mathrm{m}$ coatings; 1 .e. very near to the Reuss assumption. This can be explaned by considering the fact that the measurement takes place very near to the surface of the samples. The grains at the surface can behave more independently from each other than those lying deeper in the material.

It is also noticed that the values calculated by the isotropic model were already a rather good estimation of the values calculated by taking into account the influence of texture components. So for the investi- 
gated $\mathrm{Ni}$ coatings, it can be concluded that this influence is rather weak.

Remark.

Eq. (1) has to be solved for three unknowns: $\sigma_{11}, \sigma_{22}$ and $\delta$ when one neglects the condition: $\sigma_{11}=\sigma_{22}$, The amount of measured data points should then be increased in order to make an accurate stress analysis. This can be accomplished by measuring all diffracting (hkl) planes at more than one angle $\varphi$. This was done for the Ni $2 \mu \mathrm{m}$ coating at $\varphi=$ $0^{\circ}, 45^{\circ}$ and $90^{\circ}$. The residual stresses were than found to be: $\sigma_{11}=$ $287 \pm 26 \mathrm{MPa}$ and $\sigma_{22}=293 \pm 29 \mathrm{MPa}$ according to Reuss. From these results it can be concluded that the assumption that both stresses are equal, is very close to reality (Segmüller and Murakami [5]).

\section{CONCLUSIONS.}

A new method for residual stress analysis in very thin textured coatings has been developed. This method uses a small and constant angle between the incident X-rays and the surface of the sample. Under these conditions it is absolutely necessary to correct for eventually existing instrumental aberrations, by measuring also a powder sample.

A method is also proposed to measure pole figures under the same 'glance angle' conditions as the residual stress measurements. These polefigures have to be transformed to the normal axes system for O.D.F. analysis. Software is being developed to correct for the incompleteness of these transformed polefigures.

Measurements made on some $\mathrm{Ni}$ coatings deposited on a $\mathrm{Cu}$ substrate, show that the influence of texture in these layers is rather low. It is also shown that for these materials the assumption of equal principal stresses is valid. Strongly textured materials such as TiN will be investigated in the future.

\section{ACKNOWLEDGEMENT.}

This work has been possible thanks to a scholarship from the 'Instituut ter aanmoediging van het Wetenschappelijk Onderzoek in Nijverheid en Landbouw' (I.W.O.N.L.) - Brussels.

\section{REFERENCES.}

1. L. De Buyser, P. Van Houtte, E. Aernoudt, Proc. Tagung Eigenspannungen Darmstadt, (1990) (to be published).

2. H.J. Bunge, Texture Analysis in Materials Science: Mathematical methods, (Butterworths, London 1982).

3. W. Serruys, P. Van Houtte, E.Aernoudt, Residual Stresses in Science and Technology, (DGM, Oberursel 1986).

4. W. Serruys, Röntgenografisch Meten van Restspanningen in Getextureerd Materiaal, (Dept. MTM K.U.Leuven, Leuven 1988).

5. A. Segmüller, Murakami, Treatise on Mat. Science and Techn. 27,143 (1988). 